\title{
Surface plasmon polaritons in dielectric/metal/dielectric structures: metal layer thickness influence
}

\author{
Kostrobij P., Polovyi V. \\ Lviv Polytechnic National University, \\ 12 S. Bandera Str., 79013, Lviv, Ukraine
}

(Received 20 March 2019; Revised 25 May 2019; Accepted 6 June 2019)

\begin{abstract}
A model is proposed and studied that makes it possible to explain experimental data on a metal layer thickness influence on the spectrum of SPPs waves in heterogeneous dielectric/metal/dielectric structures.
\end{abstract}

Keywords: surface plasmon, plasmon spectrum, metal layer thickness, dielectric permittivity.

2000 MSC: $78 \mathrm{~A} 50,78-05,78 \mathrm{~A} 25$

UDC: $537.5 .8: 535.5: 519.6$

DOI: $10.23939 / \mathrm{mmc} 2019.01 .109$

\section{Introduction}

Surface plasmon polaritons (SPPs) are collective excitations of electrons, propagating at the interface between a metal and a dielectric $[1,2]$. They are used to manipulate electromagnetic energy at the subwavelength scales, which necessitates the study of their characteristics.

A considerable amount of research on the study of SPPs spectrum in heterogeneous dielectric/metal/dielectric structures has been published for today (see bibliography in $[1,3]$ ), in which a metal layer is mainly considered as $2 \mathrm{D}$ metal or metallic graphene using the corresponding characteristic expressions for the dielectric function $\varepsilon(\boldsymbol{q}, \omega)\left(\boldsymbol{q}=\left(k_{x}, k_{y}\right)\right.$ is $2 \mathrm{D}$ vector, $\omega$ is frequency) of a metal.

In the case when a metal layer is a 3D structure, the Drude model is widely used to describe SPPs $[1,4]$ in which the dielectric permittivity of a metal is expressed by the formula:

$$
\begin{gathered}
\varepsilon\left(\boldsymbol{q}, z, z^{\prime}, \omega\right)=\varepsilon^{D}(\omega) \delta\left(z-z^{\prime}\right), \\
\varepsilon^{D}(\omega)=1-\frac{\omega_{p}^{2}}{\omega^{2}+i \gamma \omega} .
\end{gathered}
$$

Unfortunately, this approach to the description of SPPs does not allow taking into account the influence of the thickness of a metal layer and size effects on their properties.

Recently, in the paper [5] there has been presented the results of experimental studies on the influence of a thickness of a metal film on the spectrum $\hbar \omega$ ( $\hbar$ is the reduced Planck constant [6]) of SPPs, where it has been demonstrated that such a dependence is significant in the area of small $(\sim 1-5 \mathrm{~nm})$ thicknesses. In this paper, a mathematical model for $\varepsilon\left(\boldsymbol{q}, \omega, z, z^{\prime}\right)$ is proposed and studied and it is shown that taking into account the thickness of a metal layer can be described by such a model; and the obtained results qualitatively coincide with the experimental results.

\section{Problem formulation}

Consider a heterogeneous structure (whose geometry is depicted in Fig. 1) formed by two nonconducting media with dielectric permittivities $\varepsilon_{1}$ and $\varepsilon_{3}$ between which a metal nanofilm with thickness $L$ is sandwiched. We assume that dielectric permittivities $\varepsilon_{1}$ and $\varepsilon_{3}$ are functions of the time variable, i.e.

$$
\varepsilon_{1}=\varepsilon_{1}(t), \quad \varepsilon_{3}=\varepsilon_{3}(t) .
$$


Dielectric permittivity of metal is $\varepsilon_{2}\left(\boldsymbol{r}, \boldsymbol{r}^{\prime}, t\right)$ and for the geometry of a dielectric/metal/dielectric heterostructure has the form

$$
\varepsilon_{2}=\varepsilon_{2}\left(\boldsymbol{r}_{\|}-\boldsymbol{r}_{\|}^{\prime}, z, z^{\prime}, t\right), \quad \boldsymbol{r}_{\|}=(x, y) .
$$

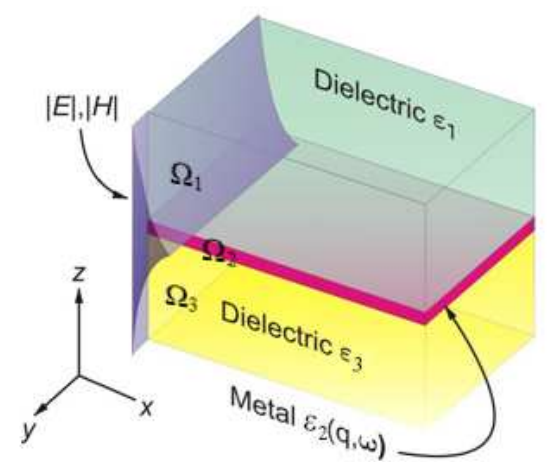

Fig. 1. Schematic representation of dielectric/metal/dielectric structure.

Let us consider a problem of describing the propagation of electromagnetic waves, which are localized at the interface between a dielectric $(z \geqslant L)$ and a metal $(0<z<L)$. These waves are called surface plasmons [1]. A mathematical model describing the propagation of surface plasmons is based on the Maxwell's equations system [1,2]:

$$
\begin{gathered}
\nabla \cdot \boldsymbol{D}=\rho, \quad \nabla \cdot \boldsymbol{B}=0, \\
\nabla \times \boldsymbol{E}=\rho, \quad \nabla \times \boldsymbol{H}=\boldsymbol{J}+\frac{\partial \boldsymbol{D}}{\partial t},
\end{gathered}
$$

where $\boldsymbol{D}$ is electric flux density, $\boldsymbol{B}$ is magnetic flux density, $\boldsymbol{E}$ is electric field strength, $\boldsymbol{H}$ is magnetic field strength, $\rho$ is electric charge density and $\boldsymbol{J}$ is electric current density. We assume that external charges $\rho$ in the area of contact between dielectrics and metal are absent, namely $\nabla \cdot \boldsymbol{D}=0$. Here "." is the dot product, " $x$ " is the cross product.

We assume that interconnection between the vectors $\boldsymbol{E}$ and $\boldsymbol{D}[2]$, namely

$$
\boldsymbol{D}\left(\boldsymbol{r}_{\|}, z, t\right)=\iiint d \boldsymbol{r}_{\|}^{\prime} d z^{\prime} d t^{\prime} \varepsilon_{i}\left(\boldsymbol{r}_{\|}-\boldsymbol{r}_{\|}^{\prime}, z, z^{\prime}, t-t^{\prime}\right) \boldsymbol{E}\left(\boldsymbol{r}_{\|}^{\prime}, z^{\prime}, t^{\prime}\right), \quad i=1,2,3 .
$$

Let us write the system of Maxwell's equations (4) in Fourier variables. We will define the Fourier transform with respect to time as

$$
f(t)=\frac{1}{2 \pi} \int_{-\infty}^{\infty} \tilde{f}(\omega) e^{i \omega t} d \omega, \quad \tilde{f}(\omega)=\int_{-\infty}^{\infty} f(t) e^{-i \omega t} d t .
$$

And $\varepsilon_{i}\left(\boldsymbol{r}_{\|}-\boldsymbol{r}_{\|}^{\prime}, z, z^{\prime}, t-t^{\prime}\right)$ is expressed by the following equation:

$$
\varepsilon_{i}\left(\boldsymbol{r}_{\|}-\boldsymbol{r}_{\|}^{\prime}, z, z^{\prime}, t-t^{\prime}\right)=\frac{\Omega}{(2 \pi)^{3}} \int_{-\infty}^{\infty} d \omega \int_{\Omega} d \boldsymbol{q} \varepsilon_{i}\left(\boldsymbol{q}, z, z^{\prime}, \omega\right) e^{-i\left(\boldsymbol{q}, \boldsymbol{r}_{\|}-\boldsymbol{r}_{\|}^{\prime}\right)-i \omega\left(t-t^{\prime}\right)}
$$

where $\Omega$ is the domain of the $2 \mathrm{D}$ vector $\boldsymbol{q}=\left(k_{x}, k_{y}\right)$.

We will assume that

$$
\begin{aligned}
& \varepsilon_{1}\left(\boldsymbol{r}_{\|}-\boldsymbol{r}_{\|}^{\prime}, z, z^{\prime}, t-t^{\prime}\right)=\varepsilon_{1}\left(t-t^{\prime}\right) \delta\left(\boldsymbol{r}_{\|}-\boldsymbol{r}_{\|}^{\prime}\right) \delta\left(z-z^{\prime}\right), \\
& \varepsilon_{2}\left(\boldsymbol{r}_{\|}-\boldsymbol{r}_{\|}^{\prime}, z, z^{\prime}, t-t^{\prime}\right)=\varepsilon_{2}\left(\boldsymbol{r}_{\|}-\boldsymbol{r}_{\|}^{\prime}, z, z, t-t^{\prime}\right) \delta\left(z-z^{\prime}\right), \\
& \varepsilon_{3}\left(\boldsymbol{r}_{\|}-\boldsymbol{r}_{\|}^{\prime}, z, z^{\prime}, t-t^{\prime}\right)=\varepsilon_{3}\left(t-t^{\prime}\right) \delta\left(\boldsymbol{r}_{\|}-\boldsymbol{r}_{\|}^{\prime}\right) \delta\left(z-z^{\prime}\right),
\end{aligned}
$$

where $\delta\left(z-z^{\prime}\right)$ is the Dirac delta function [6].

The polarization of the waves corresponds to the transverse magnetic (TM) mode for the vectors $\boldsymbol{E}$ and $\boldsymbol{H}$, that is

$$
\boldsymbol{E}=\left(E_{x}, 0, E_{y}\right), \quad \boldsymbol{H}=\left(0, H_{y}, 0\right) .
$$

Consequently [1], the magnetic field propagates along the axis $O X$ and is homogeneous along the axis $O Y$

$$
\boldsymbol{H}(\boldsymbol{r}, \omega)=\boldsymbol{H}(z, \omega) e^{i k_{x} x},
$$

$k_{x}$ is a wave vector in the direction of propagation. 
For $\boldsymbol{H}$ we obtain a system of wave equations for all the domains of the heterogeneous structure [1]:

$$
\begin{aligned}
& \frac{\partial^{2} H_{y}(z, \omega)}{\partial z^{2}}+\left(k_{0}^{2} \varepsilon_{1}(\omega)-k_{x}^{2}\right) H_{y}(z, \omega)=0, \\
& \frac{\partial^{2} H_{y}(z, \omega)}{\partial z^{2}}+\left(k_{0}^{2} \varepsilon_{2}(\boldsymbol{q}, z, z, \omega)-k_{x}^{2}\right) H_{y}(z, \omega)=0, \\
& \frac{\partial^{2} H_{y}(z, \omega)}{\partial z^{2}}+\left(k_{0}^{2} \varepsilon_{3}(\omega)-k_{x}^{2}\right) H_{y}(z, \omega)=0,
\end{aligned}
$$

where $k_{0}=\omega / c$. In order to solve the system (11)-(13), we need to find out expressions for dielectric permittivities $\varepsilon_{1}(\omega), \varepsilon_{2}(\boldsymbol{q}, z, z, \omega)$, and $\varepsilon_{3}(\omega)$.

\section{Model of dielectric permittivity of a metal layer}

Here and subsequently, we will use a high-frequency approximation for dielectric layers, this implies that in (8) the first and the last expressions can be rewritten as follows

$$
\begin{gathered}
\varepsilon_{1}(\omega)=\varepsilon_{1}(\infty)=\varepsilon_{1}=\text { const } \\
\varepsilon_{3}(\omega)=\varepsilon_{3}(-\infty)=\varepsilon_{3}=\text { const. }
\end{gathered}
$$

As a model for the dielectric function $\varepsilon_{2}\left(\boldsymbol{q}, z, z^{\prime}, \omega\right)$ of the metal layer, we will use the diagonal component of the dielectric permittivity tensor of a metal film which is obtained in [7],

$$
\varepsilon\left(\boldsymbol{r}, \boldsymbol{r}^{\prime}, \omega\right)=\left(1-\frac{\omega_{p}^{2}}{n_{e} \omega^{2}} \sum_{n} f_{n}\left|\psi_{n}\left(\boldsymbol{r}^{\prime}\right)\right|^{2}\right) \delta\left(\boldsymbol{r}-\boldsymbol{r}^{\prime}\right) .
$$

Here $\omega_{p}=\sqrt{4 \pi n_{e} e^{2} / m_{e}}$ is the plasma frequency [1,4], $n_{e}$ is an electron density in a metal, $f_{n}=$ $\Theta\left(\varepsilon_{n}-\varepsilon_{F}\right)$ is the Fermi-Dirac function [7,8], $\Theta(x)$ is the Heaviside step function [8], $\varepsilon_{F}$ is the Fermi energy $[4,8], \boldsymbol{r}=\left(\boldsymbol{r}_{\|}, z\right)$.

The function

$$
\psi_{n}(x, y, z)=\sqrt{\frac{2}{S}} e^{i\left(\boldsymbol{q} \cdot \boldsymbol{r}_{\|}\right)} \phi_{n}(z)
$$

is a wave function [6] of an electron in the metal layer and $\phi_{n}(z)$ is the solution of equation

$$
-\frac{\hbar^{2}}{2 m} \frac{d^{2}}{d z^{2}} \phi_{n}(z)+U(z) \phi_{n}(z)=W \phi_{n}(z)
$$

which is the Schrödinger equation [3] that describes behaviour of an electron in a metal film $[6,9,10]$. Potential $U(z)$ that simulates surfaces bounding the film has the form

$$
U(z)=\left\{\begin{array}{lll}
U_{1} & \text { if } \quad z<0, \\
0 & \text { if } \quad 0<z<L, \\
U_{2} & \text { if } \quad z>L,
\end{array}\right.
$$

where $L$ is the film thickness. The solutions of the equation (17) that satisfies the conditions $\phi(z \rightarrow \pm \infty) \rightarrow 0$ can be presented as follows

$$
\phi_{n}(z)=\left\{\begin{array}{lll}
A e^{\chi_{1} z}, \quad \chi_{1}=\sqrt{\frac{2 m}{\hbar^{2}}\left(U_{1}-W\right)} & \text { if } \quad z<0, \\
C_{1} e^{i k z}+C_{2} e^{-i k z}, \quad k=\sqrt{\frac{2 m}{\hbar^{2}} W} & \text { if } \quad 0<z<L, \\
B e^{-\chi_{1} z}, \quad \chi_{2}=\sqrt{\frac{2 m}{\hbar^{2}}\left(U_{2}-W\right)} \quad \text { if } \quad z>L .
\end{array}\right.
$$

Constants $A, C_{1}, C_{2}$, and $B$ we will determine using continuity conditions for $\phi_{n}(z)$ and $\frac{d \phi_{n}(z)}{d z}$ on the boundaries $z=0$ and $z=L$ and a normalization condition 


$$
\int_{-\infty}^{\infty}\left|\phi_{n}(z)\right|^{2}=1
$$

which, actually, yields the condition $\phi(z \rightarrow \pm \infty) \rightarrow 0$. Hence, the expressions for constants have the form

$$
\begin{aligned}
C_{1}= & A\left(\frac{1}{2}-\frac{i \chi_{1}}{2 k}\right), \quad C_{2}=A\left(\frac{1}{2}+\frac{i \chi_{1}}{2 k}\right) . \\
B= & A\left(\cos (k L)+\frac{\chi_{1}}{k} \sin (k L)\right) e^{\chi_{2} L} \\
|A|^{2}= & \left(\frac{1}{2 \chi_{1}}+\frac{1}{2 \chi_{2}}\left(\cos (k L)+\frac{\chi_{1}}{k} \sin (k L)\right)^{2}\right. \\
& \left.+\frac{L}{2}\left(\frac{\chi_{1}^{2}}{k^{2}}+1\right)+\frac{\chi_{1}}{2 k^{2}}(1-\cos (2 k L))+\frac{1}{4}\left(\frac{1}{k}-\frac{\chi_{1}^{2}}{k^{3}}\right) \sin (2 k L)\right)^{-1} .
\end{aligned}
$$

In order to find $k$, we need to solve the following equation $[3,9,10]$

$$
k L=\pi n-\left(\arcsin \frac{k \hbar}{\sqrt{2 m U_{1}}}+\arcsin \frac{k \hbar}{\sqrt{2 m U_{2}}}\right),
$$

the roots of (24) will determine a value of

$$
W_{n}=\frac{\hbar^{2} k_{n}^{2}}{2 m}
$$

which is discrete.

The maximum number of energy levels $n_{\max }$ we determine from the condition

$$
\begin{gathered}
n_{\max }=\left[\frac{1}{\pi}\left(L \min \left(S_{1}, S_{2}\right)+\arcsin \frac{\min \left(S_{1}, S_{2}\right)}{S_{1}}+\arcsin \frac{\min \left(S_{1}, S_{2}\right)}{S_{2}}\right)\right], \\
S_{i}=\sqrt{\frac{2 m}{\hbar^{2}} U_{i}}, \quad i=1,2 .
\end{gathered}
$$

Square brackets indicate taking the integer part.

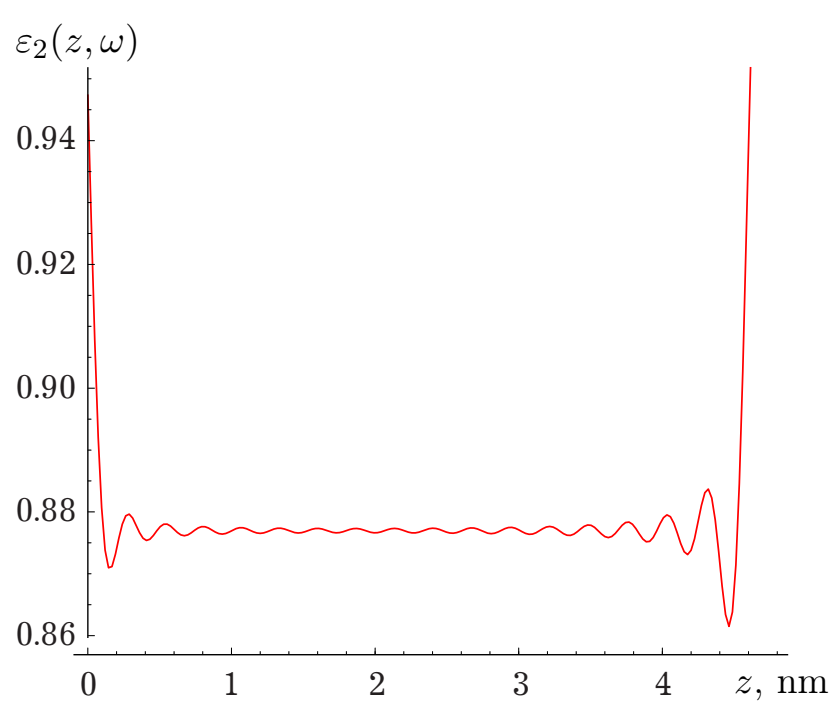

Fig. 2. The dielectric permittivity of the metal layer $(L=5 \mathrm{~nm})$ when $\frac{\omega}{\omega_{p}}=4$.
Expression for $\varepsilon\left(\boldsymbol{q}, z, z^{\prime}, \omega\right)$ (7) for the model (15) has the form (for details see [7])

$$
\begin{gathered}
\varepsilon_{2}\left(0, z, z^{\prime}, \omega\right)=\varepsilon_{2}(z, \omega) \delta\left(z-z^{\prime}\right) \\
=\left(1-\frac{\omega_{p}^{2}}{\pi n_{e} \omega^{2}} \sum_{n=1}^{n_{\max }}\left(k_{F}^{2}-\right.\right. \\
\left.\left.\quad k_{n}^{2}\right)\left|\phi_{n}(z)\right|^{2}\right) \\
\times \delta\left(z-z^{\prime}\right) .
\end{gathered}
$$

The results of numerical calculations of $\varepsilon_{2}(z, \omega)$ for specific values $U_{1}=4.2 \mathrm{eV}$ and $U_{2}=$ $5 \mathrm{eV}$, which correspond to the dielectrics $(1-$ polyethylene, $2-\mathrm{SiO}_{2}$ ), are shown in Fig. 2.

The results obtained have shown that the dielectric function $\varepsilon_{2}(z, \omega)$ is different from constant only near the contact areas $(z=L$ and $z=0$ ) (Fig. 2). This allows making some simplifications when studying the system (11)-(13). 


\section{Investigation of the influence of the thickness of metal film on the wave spectrum}

To solve the system of wave equations (11)-(13), Eq. (27) will be assumed that

$$
\varepsilon_{2}\left(z, z^{\prime}, \omega\right)=\left(\varepsilon_{2}(L, \omega)+\alpha \Delta \varepsilon_{2}(z, \omega)\right) \delta\left(z-z^{\prime}\right),
$$

where

$$
\begin{aligned}
\varepsilon_{2}(L, \omega) & =\frac{1}{L} \int_{0}^{L} \varepsilon_{2}(z, \omega) d z=1-\frac{\omega_{p}^{2}}{2 \pi n_{e} \omega^{2}} \sum_{n=1}^{n_{\max }}\left(k_{F}^{2}-k_{n}^{2}\right){\overline{\left|\phi_{n}(z)\right|^{2}}}^{2} \\
{\overline{\left|\phi_{n}(z)\right|^{2}}}^{2} & =\frac{1}{L} \int_{0}^{L}\left|\phi_{n}(z)\right|^{2} d z \\
& =|A|^{2}\left(\frac{1}{2}\left(\frac{\chi_{1}^{2}}{k^{2}}+1\right)+\frac{\chi_{1}}{2 k^{2} L}(1-\cos (2 k L))+\frac{1}{4 L}\left(\frac{1}{k}-\frac{\chi_{1}^{2}}{k^{3}}\right) \sin (2 k L)\right) .
\end{aligned}
$$

Substitution of (29) into (12) yields

$$
\frac{\partial^{2} H_{y}(z, \omega)}{\partial z^{2}}+\left(k_{0}^{2}\left(\varepsilon_{2}(L, \omega)+\alpha \Delta \varepsilon_{2}(z, \omega)\right)-k_{x}^{2}\right) H_{y}(z, \omega)=0 .
$$

The solution of the equation $(31)$ for $H_{y}(z, \omega)$ can be found as expansion into a series in increasing powers of $\alpha$

$$
H_{y}(z, \omega)=\sum_{m=0}^{\infty} \alpha^{m} H_{y, m}(z, \omega) .
$$

In particular, the first two equations $H_{y, 0}(z, \omega)$ and $H_{y, 1}(z, \omega)$ are following:

$$
\begin{aligned}
& \frac{\partial^{2} H_{y, 0}(z, \omega)}{\partial z^{2}}+\left(k_{0}^{2} \varepsilon(L, \omega)-k_{x}^{2}\right) H_{y, 0}(z, \omega)=0, \\
& \frac{\partial^{2} H_{y, 1}(z, \omega)}{\partial z^{2}}+\left(k_{0}^{2} \varepsilon(L, \omega)-k_{x}^{2}\right) H_{y, 1}(z, \omega)=-k_{0}^{2} \alpha \Delta \varepsilon_{2}(z, \omega) H_{y, 0}(z, \omega) .
\end{aligned}
$$

When modeling the influence of the thickness of a metal film $L$ on SPPs spectrum, we will limit ourselves to the case $H_{y}(z, \omega) \approx H_{y, 0}(z, \omega)$. In this case, the dispersion relation has the form

$$
\begin{gathered}
e^{-4 k_{1} \frac{L}{2}}=\frac{k_{1} / \varepsilon_{1}+k_{2} / \varepsilon_{2}}{k_{1} / \varepsilon_{1}+k_{2} / \varepsilon_{2}} \frac{k_{3} / \varepsilon_{1}+k_{3} / \varepsilon_{2}}{k_{3} / \varepsilon_{3}+k_{2} / \varepsilon_{2}}, \\
k_{i}^{2}=k_{x}^{2}-k_{0}^{2} \varepsilon_{i}, \quad i=1,2,3 ; \quad k_{0}=\frac{\omega}{c},
\end{gathered}
$$

which coincides with the results obtained in Ref. [1]. Here $\varepsilon_{1}=\varepsilon(\omega), \varepsilon_{2}=\varepsilon(L, \omega)$, and $\varepsilon_{3}=\varepsilon(\omega)$.

Similarly as in our previous work [11], for the upper layer we took a polyethylene with a permittivity constant $\varepsilon_{1}=2.3$ and the electron work function $U_{1}=4.24 \mathrm{eV}$; $\mathrm{SiO}_{2}$ for the lower dielectric substrate with a permittivity constant $\varepsilon_{1}=4$ and the electron work function $U_{2}=5 \mathrm{eV}$.

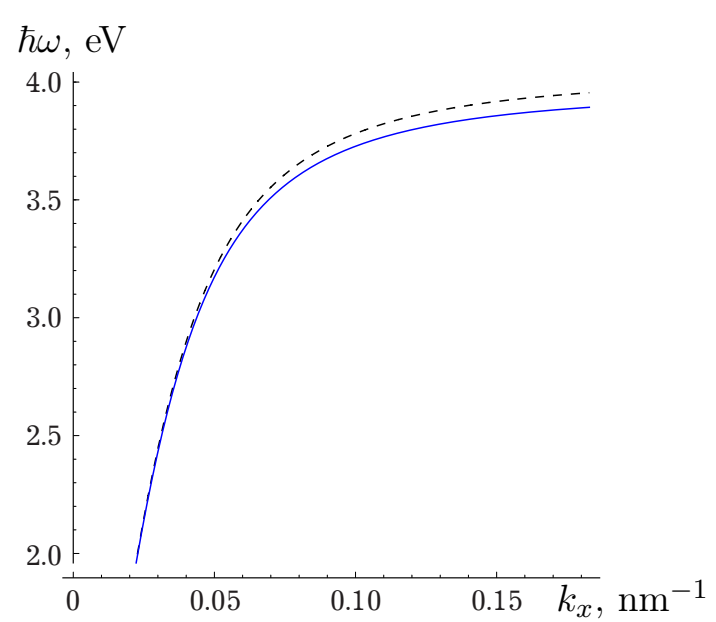

Fig. 3. SPPs spectrum for the Drude model $\varepsilon^{D}(\omega)$ (dotted line) and $\varepsilon_{2}(L, \omega)$ (solid line). The dielectric function of the metal layer (gold) is described by the function (16)

$$
\varepsilon_{2}(L, \omega)=1-\frac{\omega_{p}^{2}}{2 \pi n_{e} \omega^{2}} \sum_{n=1}^{n_{\max }}\left(k_{F}^{2}-k_{n}^{2}\right){\overline{\left|\phi_{n}(z)\right|^{2}}}^{2} .
$$


Fig. 3 shows the result of spectrum calculations obtained from (35) using (29), (30). These results we compared to the data obtained for the Drude model with negligible damping [1]

$$
\varepsilon(L, \omega)=\varepsilon^{D}(\omega)=1-\frac{\omega_{p}^{2}}{\omega}
$$

in which the plasmon frequency spectrum does not depend on the thickness $L$.

In Table 1 the results of numerical calculations of the dependence of frequency $\omega^{*}=\frac{\omega}{\omega_{p}}$, and a wave vector $k_{x}^{*}=\frac{k_{x} c}{\omega_{p}}$ on the number of levels of dimensional quantization $n_{\max }$ are provided [12].

Table 1.

\begin{tabular}{|c|c|c|c|}
\hline & $\varepsilon_{2}\left(L, \omega^{*}\right)$ & $\varepsilon^{D}\left(\omega^{*}\right)$ & \\
\hline$L(\mathrm{~nm})$ & $\omega^{*}$ & $\omega^{*}$ & $n_{\max }$ \\
\hline \multicolumn{4}{|c|}{$k_{x}^{*}=1$} \\
\hline 100 & 0.33992328 & 0.34237082 & 335 \\
\hline 1000 & 0.34016832 & 0.34237082 & 3357 \\
\hline 5000 & 0.34019295 & 0.34237082 & 16789 \\
\hline 10000 & 0.34019603 & 0.34237082 & 33579 \\
\hline 30000 & 0.34019749 & 0.34237082 & 100737 \\
\hline \multicolumn{5}{|c|}{$k_{x}^{*}=2$} \\
\hline 100 & 0.40974355 & 0.41421356 & 335 \\
\hline 1000 & 0.41021317 & 0.41421356 & 3357 \\
\hline 5000 & 0.41025793 & 0.41421356 & 16789 \\
\hline 10000 & 0.41026353 & 0.41421356 & 33579 \\
\hline 30000 & 0.41026618 & 0.41421356 & 100737 \\
\hline \multicolumn{5}{|c|}{$k_{x}^{*}=3$} \\
\hline 100 & 0.42679347 & 0.43187178 & 335 \\
\hline 1000 & 0.42732626 & 0.43187178 & 3357 \\
\hline 5000 & 0.42737706 & 0.43187178 & 16789 \\
\hline 10000 & 0.42738341 & 0.43187178 & 33579 \\
\hline 30000 & 0.42738642 & 0.43187178 & 100737 \\
\hline \multicolumn{5}{|c|}{$k_{x}^{*}=4$} \\
\hline 100 & 0.43313236 & 0.43844718 & 335 \\
\hline 1000 & 0.43368968 & 0.43844718 & 3357 \\
\hline 5000 & 0.43374282 & 0.43844718 & 16789 \\
\hline 10000 & 0.43374947 & 0.43844718 & 33579 \\
\hline 30000 & 0.43375261 & 0.43844718 & 100737 \\
\hline
\end{tabular}

The data in the table show that difference between spectra $\omega\left(k_{x}\right)$ for both models increases with increasing of the wave vector $k_{x}$. Also, it should be noted that with increasing of $L, \omega\left(k_{x}\right)$ is steadily approaching from below the values obtained for the Drude model.

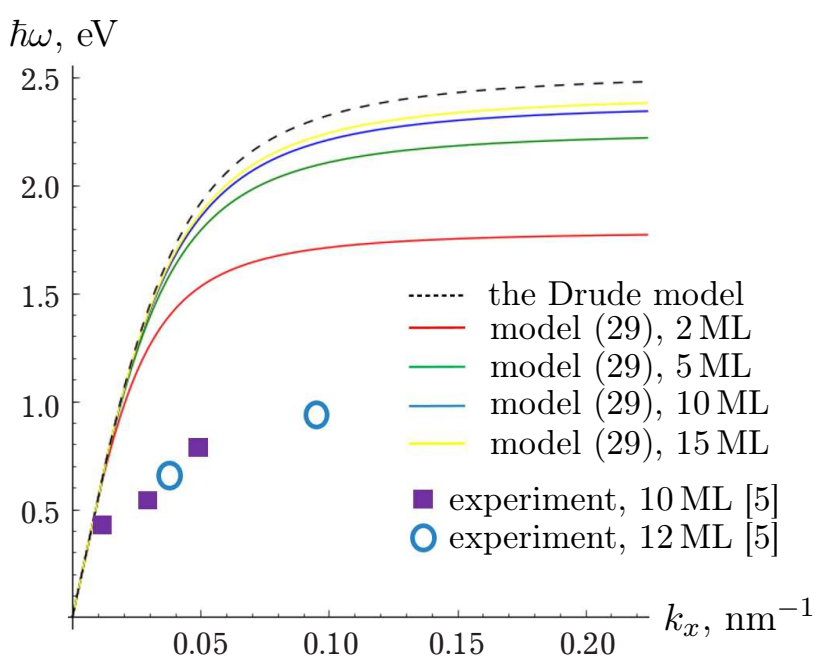

Fig. 4. SPPs spectrum for the Drude model $\varepsilon^{D}(\omega)$ (dotted line) and $\varepsilon_{2}(L, \omega)$ for the different thicknesses of a metal layer (solid lines), $1 \mathrm{ML} \sim 0.24 \mathrm{~nm}$.

In Fig. 4, the results of calculations for the structure "Si-silver- $\mathrm{SiO}_{2}$ " are shown. The dielectrics were simulated for the following parameters: $U_{1}=5 \mathrm{eV}, \varepsilon_{1}=2.4$ and $U_{1}=4.8 \mathrm{eV}, \varepsilon_{1}=11.7$ for Si and $\mathrm{SiO}_{2}$ correspondingly [1].

The same figure shows the experimental results for the structure " $\mathrm{Si}$-silver- $\mathrm{SiO}_{2}$ " published in [5]. These results demonstrate that the spectrum of plasmons strongly depends on the thickness of a metal layer when $L \sim 50 \mathrm{ML}$. As can be seen from Fig. 4, the proposed approach gives a qualitative explanation of the influence of a metal film on the SPPs spectrum.

Note that the results obtained in [5] for the SPPs spectrum were obtained for structures that consist of 2-15 monolayers, thus simulation of $\varepsilon_{2}\left(\boldsymbol{q}, z, z^{\prime}, \omega\right)$ should be carried out for such thicknesses. In such metal structures, quantum effects become significant $[9,10,13]$ and they should be taken into account.

[1] Maier S. A. Plasmonics: Fundamentals and Application. Springer, New York (2007).

[2] Jackson J. D. Classical Electrodynamics. John Wiley \& Sons (2007).

[3] Landau L. D., Bell J. S., Kearsley M. J, Pitaevskii L.P, Lifshitz E. M., Sykes J. B. Electrodynamics of Continuous Media. Elsevier, Vol. 8 (2013). 
[4] Ashcroft N. W., Mermin N. D. Solid State Physics. Harcourt College Publishers (1976).

[5] Zakaria M. Abd El-Fattah et al. Plasmonics in atomically-thin crystalline silver films. arXiv:1901.07739 (2019).

[6] Vakarchuk I. O. Kvantova mekhanika. Lviv, LNU im. I. Franka (2007), (in Ukrainian).

[7] Kurbatsky V. P. Dielectric tensor of low-dimensional metal systems. J. Exp. Theor. Phys. 125 (1), 148-158 (2017).

[8] Landau L. D., Lifshitz E. M. Statistical Physics. Elsevier, Vol. 5 (2013).

[9] Kostrobij P. P., Markovych B. M. Effect of Coulomb interaction on chemical potential of metal film. Philosophical Magazine. 98 (21), 1991-2002 (2018).

[10] Kostrobij P. P., Markovych B. M. The chemical potential and the work function of a metal film on a dielectric substrate. Philosophical Magazine Letters. 99 (1), 12-20 (2019).

[11] Kostrobij P., Pavlysh V., Nevinskyi D., Polovyi V. SPP waves in "dielectric-metal-dielectric" structures: influence of exchange correlations. Math. Model. Comput. 4 (2), 184-192 (2017).

[12] Kostrobij P. P., Polovyi V. Y. Influence of the thickness of a metal nanofilm on the spectrum of surface plasmons. Proc. of the 15th International Conference on the Experience of Designing and Application of CAD Systems (CADSM), pp. 5/58-5/61.

[13] Kostrobij P. P., Markovych B. M. Effect of the film thickness on the effective electron-electron interaction in a metal film. Math. Model. Comput. 5 (1), 21-26 (2018).

\title{
Поверхневі плазмон-поляритони в структурах “діелектрик-метал-діелектрик”: вплив товщини металевого прошарку
}

\author{
Костробій П., Польовий В. \\ Національний університет "Львівська політехніка", \\ вул. С. Бандери, 12, Львів, 79013, Україна
}

\begin{abstract}
Запропоновано та досліджено модель, яка дає змогу пояснити експериментальні дані щодо впливу товщини металевого прошарку на спектр SPP хвиль у гетерогенних структурах "діелектрик-метал-діелектрик".

Ключові слова: поверхневі плазмони, спектр плазмона, повщина металевого шару, діелектрична проникність.
\end{abstract}

2000 MSC: 78A50, 78-05, 78A25

УДК: $537.5 .8: 535.5: 519.6$ 